Wolf-Rayet Phenomena in Massive Stars and Starburst Galaxies

Proceedings IAU Symposium No. 193, (C)1999 IAU

K.A. van der Hucht, G. Koenigsberger \& P.R.J. Eenens, eds.

\title{
ISO-PHOT observations of Wolf-Rayet winds
}

\author{
Mark C. Runacres ${ }^{1}$, Ronny Blomme ${ }^{1}$, Koen Vyverman ${ }^{2}$, Martin \\ Cohen $^{3}$, Claus Leitherer ${ }^{4}$, Stanley P. Owocki ${ }^{5}$, and Martin Haas ${ }^{6}$ \\ ${ }^{1}$ Royal Observatory of Belgium, B-1180 Brussel, Belgium \\ ${ }^{2}$ Vrije Universiteit Brussel, B-1050 Brussel, Belgium \\ ${ }^{3}$ Radio Astronomy Lab., Univ. of California, Berkeley, CA 94720, USA \\ ${ }^{4}$ Space Telescope Science Institute, Baltimore, MD 21218, USA \\ ${ }^{5}$ Bartol Research Institute, Univ. of Delaware, Newark, DE 19716, USA \\ ${ }^{6}$ Max-Planck Institut für Astronomie, D-69117 Heidelberg, Germany
}

Abstract. We use ISO-PHOT maps around five WN stars to derive their farinfrared fluxes. In some cases we also detect a nebula around the star.

We have observed five WN stars (WR 62, WR 78, WR 134, WR 136, and WR 138) at 60 and $90 \mu \mathrm{m}$ with ISO-PHOT. WN stars are rather faint infrared sources, superposed on a strong, structured background. To subtract the correct background, we map a 9!25 $\times 4$ !.75 region around the star. We have used the PHT32

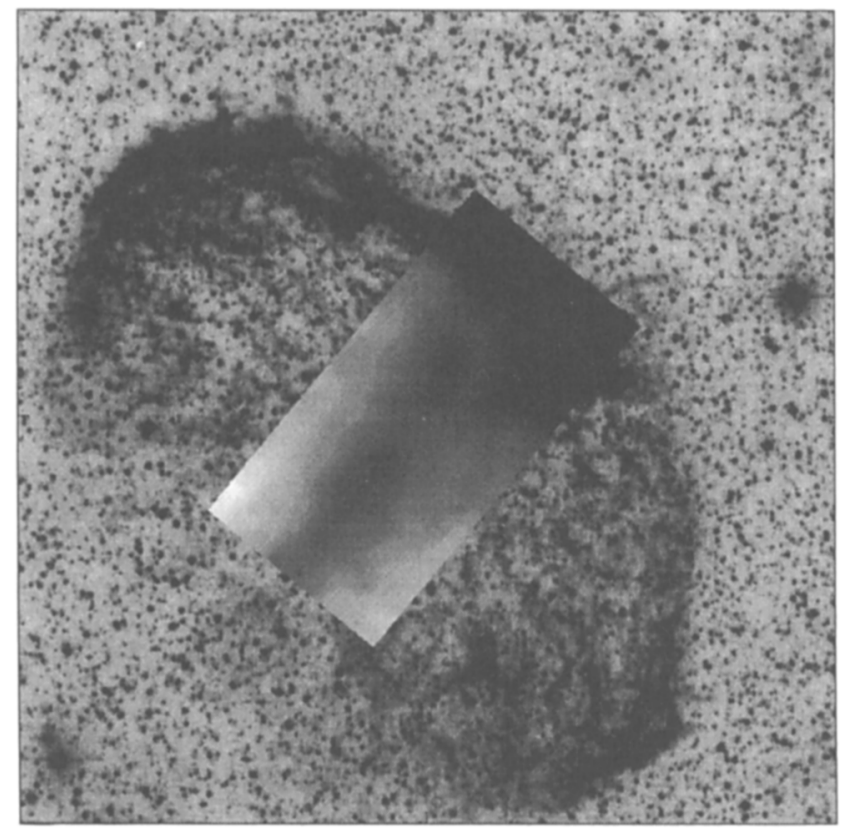

Figure 1. ISO-Рнот $60 \mu \mathrm{m}$ map around WR 136, superposed on an optical image from the Digitized Sky Survey. A part of the nebula NGC 6888 is clearly visible. The infrared morphology of the nebula is very similar to the optical one. 


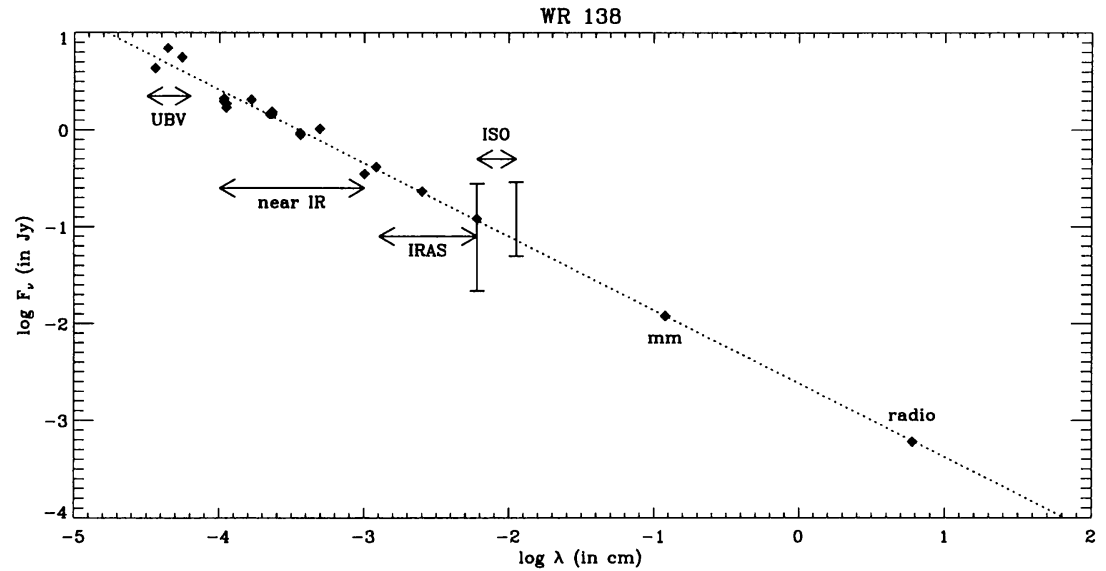

Figure 2. Preliminary fluxes for WR 138, derived from our ISO-PHOT observations. Our data are supplemented by visual, infrared, millimetre and radio data from other authors (diamonds). The dashed line is the linear best fit through the diamonds. The ISO-PHOT fluxes lie in the range of values one can estimate from the IRAS and radio data.

over-sampled mapping mode with an over-sampling factor of 3 . This reduces the pixel size from $45^{\prime \prime} \times 45^{\prime \prime}$ to $15^{\prime \prime} \times 15^{\prime \prime}$.

Besides information about the star itself, the maps in some cases show the effect of nebulosity associated with the star. We clearly see a part of the NGC 6888 nebula around WR 136: its infrared morphology is very similar to the optical morphology (see Fig. 1).

The PHT32 mapping mode has a high redundancy of information: a given point on the sky is observed repeatedly. A major problem is that these repeated determinations do not give consistent results, leading to large error bars. Preliminary results for WR 138 (see Fig. 2) and WR 78 lie in the range of values one can estimate from the IRAS and radio data. Ongoing data reduction will reduce the size of the error bars.

Acknowledgments. We are grateful to ESA-PRODEX for funding this research. 